\title{
Radial dependence of ionization losses of protons of the Earth's radiation belts
}

\author{
A. S. Kovtyukh \\ Skobeltsyn Institute of Nuclear Physics, Moscow State University, 119899, Moscow, Russia \\ Correspondence to: A. S. Kovtyukh (kovtyukhas@mail.ru)
}

Received: 25 August 2015 - Revised: 11 December 2015 - Accepted: 17 December 2015 - Published: 15 January 2016

\begin{abstract}
Coulomb losses and charge exchange of protons are considered in detail. On the basis of modern models of the plasmasphere and the exosphere, the radial dependences of the rates of ionization losses of protons, with $\mu$ from 0.3 to $10 \mathrm{keVnT}^{-1}$, of the Earth's radiation belts near the equatorial plane are calculated for quiet periods. For calculation of Coulomb losses of protons we used data of ISEE-1 satellite (protons with energy from 24 to $2081 \mathrm{keV}$ ) on $L$ from 3 to 9, data of Explorer-45 satellite (protons with energy from 78.6 to $872 \mathrm{keV}$ ) on $L$ from 3 to 5 and data of CRRES satellite (protons with energy from 1 to $100 \mathrm{MeV}$ ) on $L \leq 3$ ( $L$ is the McIlwain parameter). It is shown that with decreasing $L$ the rate of ionization losses of protons of the radiation belts is reduced; for protons with $\mu>1.2 \mathrm{keV} \mathrm{nT}^{-1}$ in a narrow region $(\Delta L \sim 0.5)$ in the district of plasmapause in this dependence may form a local minimum of the rate. We found that the dependence from $\mu$ of the boundary on $L$ between Coulomb losses and charge exchange of the trapped protons with hydrogen atoms is well approximated by the function $L_{\mathrm{b}}=4.71 \mu^{0.32}$, where $[\mu]=\mathrm{keV} \mathrm{nT}^{-1}$. Coulomb losses dominate at $L<L_{\mathrm{b}}(\mu)$, and at $L>L_{\mathrm{b}}(\mu)$ dominates charge exchange of protons. We found the effect of subtracting the Coulomb losses from the charge exchange of protons of the radiation belts at low $\mu$ and $L$, which can simulate a local source of particles.
\end{abstract}

Keywords. Magnetospheric physics (energetic particles trapped)

\section{Introduction}

The Earth's radiation belt of protons is stationary in quiet time. Only the 11-year solar cycle variations and the small seasonal variations are observed.
For protons with $E<10 \mathrm{MeV}$ on $L>2$, the belt is formed by mechanisms of radial diffusion of particles under an influence of fluctuations of magnetic and electric fields in the range of the drift periods of trapped particles, i.e. in the range from several minutes to some hours. At the same time, the first $(\mu)$ and the second ( $I$ or $K=I / p$, where $p$ is the impulse of a particle) invariants of the drift motion of particles are conserved, and the third invariant $(\Phi)$ is violated. The first invariant is associated with the giration of charged particles in a magnetic field, the second invariant is associated with the oscillations of the particles in the magnetic tube between the mirror points and the third invariant is associated with drift of the particles around the Earth in a magnetic trap.

Radial diffusion of these protons is described by the Fokker-Planck differential equation. Under certain conditions, which performed for protons of the radiation belts, the equation is reduced to the ordinary diffusion equation (e.g., Tverskoy, 1968; Roederer, 1970; Schulz and Lanzerotti, 1974). However, the main parameter of a theory of radial diffusion of this particles, $D_{L L}$, which determines the rate of transport of particles, has a rather uncertain value. In theory, the value of $D_{L L}$ varies within 1 order of magnitude (Nakada and Mead, 1965; Tverskoy, 1968, 1969; Schulz and Lanzerotti, 1974). On the other hand, the values of $D_{L L}$ obtained from experimental data for the Earth's radiation belts vary for each $L$ by 3 or more orders of magnitude (e.g., Fig. 20 in West et al. (1981) or Fig. 1 in Alinejad and Armstrong, 2006).

To extract $D_{L L}$ from the data on radiation belts, using the diffusion equation (the inverse problem), it is necessary to have the complete and reliable values of the loss rates of trapped particles. The most fully loss mechanisms are studied for protons. They depend on the distribution of cold plasma and atoms in the geomagnetic trap. 
Modern models of these distributions are the most reliable for magnetically quiet periods. In addition, with the increase of geomagnetic activity the values of $D_{L L}$ increased and the magnitude of the effect depends on $L$. Therefore, according to data including periods of magnetic activity, we can obtain only averaged $D_{L L}(L, \mu, K)$ values which depends on the choice of the data intervals and the parameters of the satellite orbits.

Finally, the values of $D_{L L}$ are most simply derived from the data obtained near the equatorial plane. Near the equatorial plane the most reliable model of the plasmasphere are also derived from the data obtained near the equatorial plane.

\section{The loss mechanisms of protons of the Earth's radiation belts}

The main loss mechanism of protons of the radiation belts during quiet periods are the ionization losses (e.g., Tverskoy, 1968; Schulz and Lanzerotti, 1974). During these periods, protons precipitated slightly and the influence of ioncyclotron and other waves on the lifetimes of protons can be neglected (e.g., Lyons and Williams, 1984).

Ionization losses of protons arise from the Coulomb interactions of protons with electrons and from the charge exchange of protons with atoms.

Here we consider only protons near the equatorial plane, with equatorial pitch angles $\alpha_{0} \approx 90^{\circ}$ (the second adiabatic invariant of such particles $K \approx 0$ ).

In quiet times distribution of the radiation belts of protons are practically stationary and describes, when $K=0$, by the following equation:

$L^{2} \frac{\partial}{\partial L}\left(\frac{D_{L L}}{L^{2}} \frac{\partial f}{\partial L}\right)=-\left(\frac{\partial f}{\partial t}\right)_{\mathrm{cc}}-\left(\frac{\partial f}{\partial t}\right)_{\mathrm{ce}}$,

where $f(L, \mu)$ is the distribution function of protons in the phase space and $D_{L L}(L, \mu)$ is the coefficient of the radial diffusion of protons. We believe that on $L>2.5$ the internal (local) sources of protons are absent. The first term on the right side of Eq. (1) describes Coulomb losses of protons, and the second term describes the charge exchange of protons with atoms. Coulomb scattering of protons by pitch angles neglected in Eq. (1) according to Schulz and Lanzerotti (1974). The functions $f$ and $D_{L L}$ in this equation refer to the particles with given values of $\mu$.

Equation (1) shows that for each $L$-shell of the stationary radiation belt update (inflow) and losses (outflow) of protons with the given values of $\mu$ is completely balanced.

For non-relativistic protons with $\alpha_{0}=90^{\circ}(K=0)$

$f(L, \mu)=k \frac{j[L, E(L, \mu)]}{E(L, \mu)}=k f^{*}(L, \mu)$,

where $j[L, E(L, \mu)]$ is the measured fluxes of protons, $M$, $p$ and $E$ is the mass, impulse and kinetic energy of protons, and

$\mu\left(\mathrm{keVnT}^{-1}\right)=\frac{E}{B_{0}(L)}=3.215 \cdot 10^{-5} L^{3} E(\mathrm{keV})$,

where $B_{0}(L)$ is the magnetic induction near equatorial plane. The values of $\mu$ were calculated here for the dipole magnetic field.

A value of the coefficient $k / 2 M$ depends on dimensions of a quantity in Eq. (2). For dimensions $[j]=\left(\mathrm{cm}^{2} \mathrm{~s} \cdot \mathrm{ster} \cdot \mathrm{keV}\right)^{-1},[E]=\mathrm{keV}$ and $[f]=\mathrm{s}^{3} \mathrm{~cm}^{-6}$ the value $k=5.447 \cdot 10^{-31}$ (Williams, 1981, p. 189). In our calculations the coefficient $k$ is reduced, and plays no role in the results. So we will use $f^{*}(L, \mu)$ instead of $f(L, \mu)$. Equation (1) is invariant under this replacement.

We will consider the protons with $\mu$ from 0.3 to $10 \mathrm{keV} \mathrm{nT}^{-1}$ (from 30 to $1000 \mathrm{MeV} \mathrm{G}^{-1}$ ) and $L$ from 2 to 10. On the geosynchronous orbit such protons have energy from $\sim 30 \mathrm{keV}$ to $\sim 1 \mathrm{MeV}$ and on $L=3$ they have energy from $\sim 350 \mathrm{keV}$ to $\sim 11.5 \mathrm{MeV}$.

\subsection{The charge exchange losses of protons}

Hydrogen atoms are dominated in the composition of the exosphere on $L>2.5$ near the equatorial plane. The contributions of other atoms (oxygen, etc.) and molecules in the charge exchange rate of trapped protons do not exceed a few percent (e.g., Cornwall, 1972). The charge exchange rate of protons of the radiation belts on the hydrogen atoms, normalized by $f^{*}(L, \mu)$, is described by the following expression:

$$
\begin{aligned}
-\frac{1}{f^{*}}\left(\frac{\partial f^{*}}{\partial t}\right)_{\mathrm{ce}} & =-\left(\frac{\partial \ln f^{*}}{\partial t}\right)_{\mathrm{ce}} \\
& =\tau_{\mathrm{ce}}^{-1}=\sigma_{\mathrm{ce}}(E) n_{\mathrm{H}}(L) v,
\end{aligned}
$$

where $\sigma_{\mathrm{ce}}(E)$ is a cross section for the charge exchange of protons with hydrogen atoms, $n_{\mathrm{H}}(L)$ is a density of the hydrogen atoms near the equatorial plane $\left(\alpha_{0}=90^{\circ}\right), v$ is a velocity of protons and $\tau_{\mathrm{ce}}$ is the lifetime of protons.

The cross sections of charge exchange of protons with $E>1 \mathrm{keV}$ on the hydrogen atoms are well studied both theoretically and experimentally (Claflin, 1970; Lindsay and Stebbings, 2005), and approximated by the following expression (Jentsch, 1984):

$\sigma_{\mathrm{ce}}=3.27 \cdot 10^{-15} \sqrt{\frac{E}{E_{\mathrm{ce}}}} \exp \left(-\sqrt{\frac{E}{E_{\mathrm{ce}}}}\right)$,

where $E_{\mathrm{ce}}=1.76 \mathrm{keV},[E]=\mathrm{keV}$ and $\left[\sigma_{\mathrm{ce}}\right]=\mathrm{cm}^{2}$. In Fig. 1 the thick curve shows the experimental dependence $\sigma_{\text {ce }}(E)$ for protons according to Claflin (1970) and crosses shows our calculations by Eq. (5).

From Fig. 1 it is seen that Eq. (5) is in good agreement with the results of Claflin (1970) for $E>70 \mathrm{keV}$, but at lower energies it deviates from them by $10-30 \%$. The deviation is maximum for $E=20 \mathrm{keV}$. 


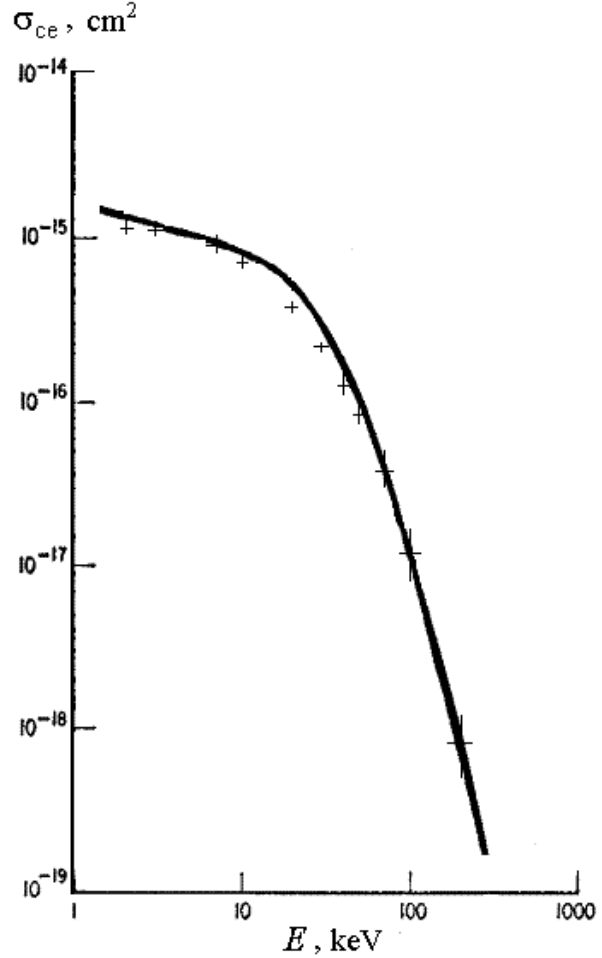

Figure 1. Comparison of the experimental dependence of $\sigma_{\mathrm{ce}}(E)$ in Claflin (1970) for protons (thick curve) with approximation (crosses) in Jentsch (1984).

Note that we do not have enough experimental data on the cross section of charge exchange of protons with $E>100 \mathrm{keV}$ and enough experimental data necessary to carry out the extrapolation, which can lead to errors much more than $30 \%$. Such an extrapolation of the experimental data (taking into account the theoretical results) was conducted in Claflin (1970) to $E=1 \mathrm{MeV}$, but for our goals it is sufficient to have reliable data on the cross sections of charge exchange of protons only to $E \sim 300 \mathrm{keV}$, as at $E>300 \mathrm{keV}$ for $\mu=0.3-10 \mathrm{keV} \mathrm{nT}^{-1}$ the Coulomb losses of protons are the main (see below, Figs. 7 and 8).

According to Cowley (1977), the density of the hydrogen atoms in the equatorial plane

$n_{\mathrm{H}}\left(\mathrm{cm}^{-3}\right)=4 \cdot 10^{4} \mathrm{~L}^{-3.5}$,

which is in good agreement with the average values of $n_{\mathrm{H}}$ in the modern models of the exosphere (e.g., Østgaard et al., 2003; Zoennchen et al., 2013).

The velocity of non-relativistic protons

$v\left(\mathrm{~cm} \cdot \mathrm{s}^{-1}\right)=4.86 \cdot 10^{7} \sqrt{E(\mathrm{keV})}$.

Taking into account Eqs. (3) and (5)-(7), the Eq. (4) transformed to

$-\left(\frac{\partial \ln f^{*}}{\partial t}\right)_{\mathrm{ce}}=\tau_{\mathrm{ce}}^{-1}=149 \cdot \mu \cdot L^{-6.5} \exp \left(-133 \sqrt{\frac{\mu}{L^{3}}}\right)$,

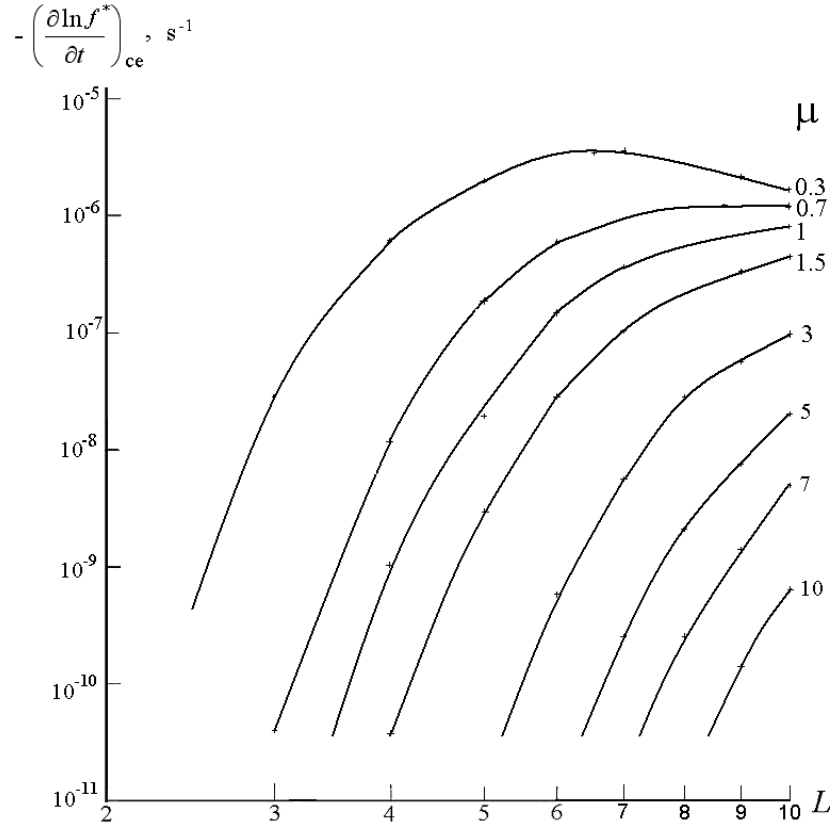

Figure 2. The radial dependences of rates of losses of protons with $\mu$ from 0.3 to $10 \mathrm{keV} \mathrm{nT}^{-1}$, on charge exchange with the atoms of the exosphere, normalized to $f^{*}(L, \mu)$.

where $[\tau]=\mathrm{s}$ and $[\mu]=\mathrm{keVnT}^{-1}$. Equation (7) has a maximum at

$L_{\mathrm{m}}=9.8 \cdot \mu^{1 / 3}$.

Figure 2 shows the radial dependences of the rate of the charge exchange loss of protons with various values of $\mu$, from 0.3 to $10 \mathrm{keV} \mathrm{nT}^{-1}$, in accordance with Eq. (8). Also it takes into account all the deviations of the experimental values of $\sigma_{\mathrm{ce}}(E)$ from Eq. (5). Calculated points between which we make extrapolations marked by the crosses.

The values of $n_{\mathrm{H}}(L)$ depend on the period of record and may deviate from accepted here the average values of $n_{\mathrm{H}}$ in 1.5-2 times (e.g., Østgaard et al., 2003; Zoennchen et al., 2013). Such deviations are the main contributors to the errors calculated here loss rate of protons, associated with their charge exchange.

\subsection{The Coulomb losses of protons}

In contrast to charge exchange when protons come out from the belts in single acts of interactions with atoms, during Coulomb losses protons gradually shift to low energy range and are replaced by protons from a higher energy range. Therefore, the Coulomb losses depend not only on the rate of loss of the individual particles, but also from the steepness of the real energy spectrum of protons at certain $L$, i.e. from the form of $f^{*}(\mu)$. And if at the given value of $\mu$ at the certain $L$ we have a positive slope of the spectrum, $\partial f^{*} / \partial \mu>0$, then the Coulomb losses do not involve reduction but an increase in density of the particles in this cell $\{\mu, L\}$. 


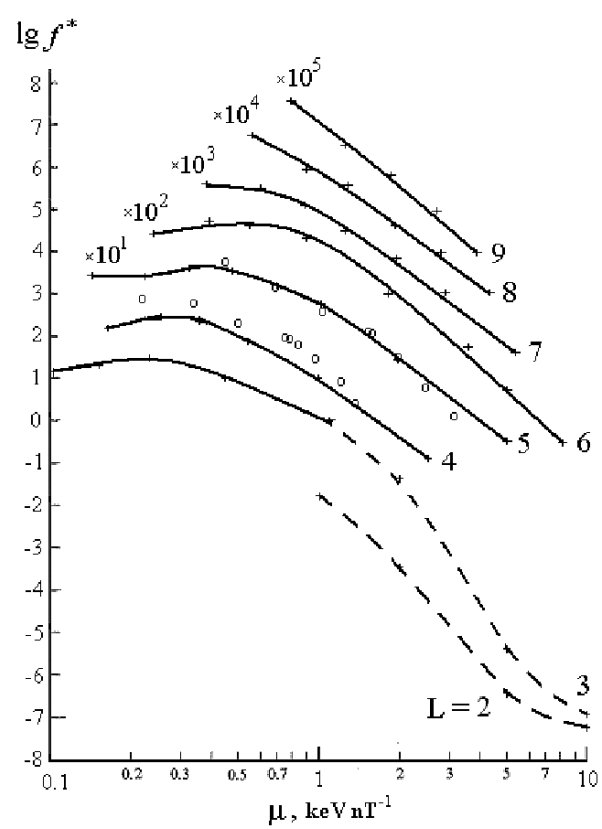

Figure 3. The functions $f^{*}(L, \mu)$, as in Eq. (2), for a quiet proton belt constructed according to Williams (1981) for ISEE-1 (thick curves), Fritz and Spjeldvik (1981) for Explorer-45 (light circles) and Albert et al. (1998) for CRRES (dotted curves).

In accordance with Schulz and Lanzerotti (1974), p. 189,

$$
\left(\frac{\partial f^{*}}{\partial t}\right)_{\mathrm{cc}}=\frac{4 \pi e^{4} / m}{\sqrt{2 \mu B^{3} / M}} N_{\mathrm{e}} \ln \Lambda \frac{\partial f^{*}}{\partial \mu}
$$

where $e$ and $m$ are the charge and the mass of electrons, $M$ is the mass of protons, $B(L)$ is a magnetic induction, $N_{\mathrm{e}}$ is an electron density in the plasmasphere, and $\ln \Lambda=25$ is the factor Debye shielding.

Substituting in Eq. (10) the numerical values of constants, we get

$$
\begin{aligned}
-\frac{1}{f^{*}}\left(\frac{\partial f^{*}}{\partial t}\right)_{\mathrm{cc}} & =-\left(\frac{\partial \ln f^{*}}{\partial t}\right)_{\mathrm{cc}}=\tau_{\mathrm{cc}}^{-1}= \\
& -4.74 \cdot 10^{-14} N_{\mathrm{e}} L^{9 / 2} F(\mu),
\end{aligned}
$$

where $\left[\tau_{\mathrm{cc}}\right]=\sec ,\left[N_{\mathrm{e}}\right]=\mathrm{cm}^{-3}$ and

$F(\mu)=\frac{1}{f^{*} \sqrt{\mu}} \frac{\partial f^{*}}{\partial \mu}$.

The value of the numerical coefficient in Eq. (11) corresponds to $[\mu]=\mathrm{keVnT}^{-1}$ and $[F]=\mathrm{keV}^{-3 / 2} \mathrm{nT}^{3 / 2}$. Function $F(\mu)$ is determined by the shape of $f^{*}(\mu)$. The function is different on different $L$.

For finding the functions $f^{*}(L, \mu)$ consider the radial profiles of the differential fluxes of protons on $L$ from 2 to 10 based on the ISEE-1 data for the quiet time period, from 20:27 UT 24 November 1977 to 01:30 UT 25 November 1977, represented by Williams (1981). In this period the

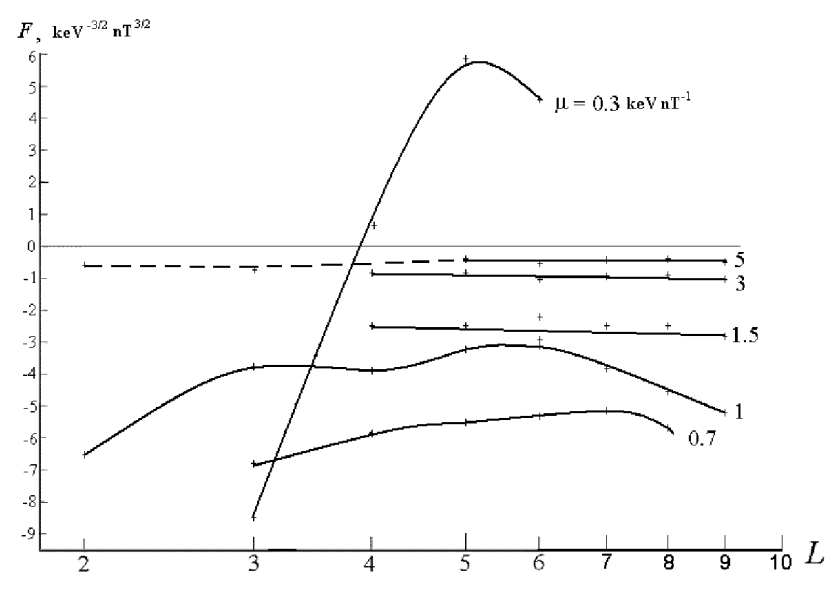

Figure 4. The functions $F(L, \mu)$, as in Eqs. (10)-(11), for the Coulomb losses of protons with $\mu$ from 0.3 to $5 \mathrm{keV} \mathrm{nT}^{-1}$.

index Dst has changed from -1 to $-3 \mathrm{nT}$, and the index $\mathrm{Kp}=0,0_{+}$and $1_{-}(\mathrm{Kp} \leq 1$ for $24 \mathrm{~h}$ prior to this period UT). The measurements carried out near the noon sector, in the eight energy channels: 24-45.5-65.3-95.5-142-210 333-849-2081 keV.

Calculations of the functions $f^{*}(L, \mu)$ were carried out in four stages (see Appendix A). Constructed by this method, according to data of the ISEE-1 (Williams, 1981) for the quiet period 24-25 November 1977, functions $f^{*}(\mu)$ for the radiation belt protons on different $L$, from 3 to 9 , shown in Fig. 3. Crosses in Fig. 3 show our calculated points between which interpolation held using the least squares method. To avoid overlapping of the curves with each other, for the spectra at $L$ from 5 to 9 are given the coefficients, that multiply the corresponding values of $f^{*}$.

Functions $f^{*}(L, \mu)$ for protons we have also calculated according to data of the Explorer-45 in Fritz and Spjeldvik (1981), averaged over 60 orbits for the quiet period 115 June 1972. The measurements of proton fluxes on this satellite were carried out on $L<5.25$ in the nine differential energy channels: $E=78.6-138.5-195.5-300 \mathrm{keV}$ and $E=363.5-375-390-430-533-674-872 \mathrm{keV}$. The results of our calculations $f^{*}(L, \mu)$ for protons with $\alpha_{0}=90^{\circ}$ on $L=4$ and 5, where the data of Explorer-45 is most reliable, are shown in Fig. 3 by the light circles. Figure 3 shows that the functions $f^{*}(L, \mu)$ calculated according to data of the ISEE-1 and Explorer-45 for quiet periods are close to each other. Note that the data from ISEE-1 and Explorer-45, used here, are in very good agreement with other data and are used in many works, including Kovtyukh $(1985,1999)$.

According to Fig. 3 and Eq. (12), we calculated the radial dependences of functions $F$. Figure 4 shows the functions for protons with $\mu$ from 0.3 to $5 \mathrm{keV} \mathrm{nT}^{-1}$. Crosses in Fig. 4 show our calculated points between which interpolation is held using the least squares method. 


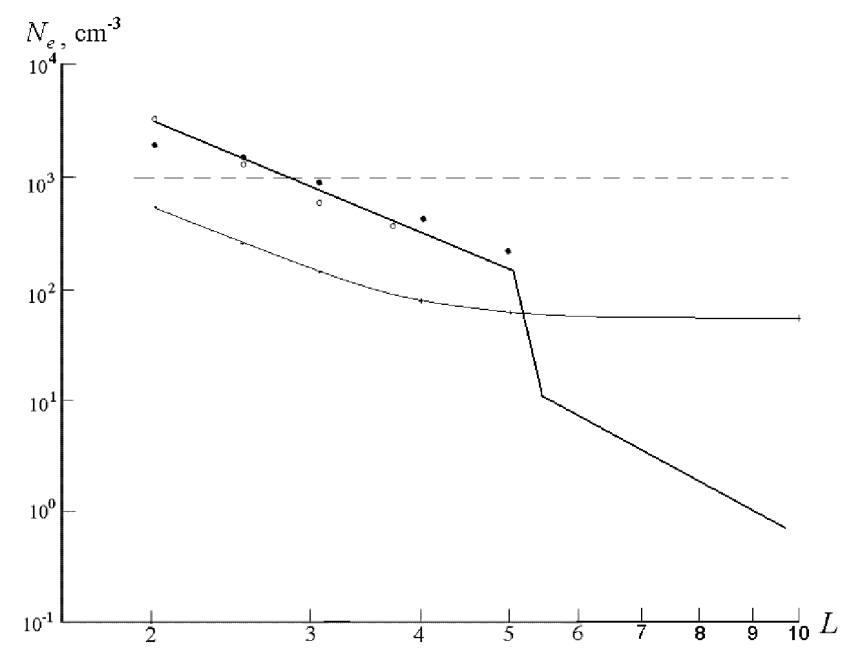

Figure 5. Averaged over the MLT the dependences $N_{\mathrm{e}}(L)$ of a density of an electron component of the cold plasma near the equatorial plane for a quiet and weakly disturbed periods according to the model of Ozhogin et al. (2012) (thick broken curve), the model of Tarsai et al. (1988) (bright spots) and the model of Carpenter and Anderson (1992) (dark dots). Thin curve is $N_{\mathrm{e}}(L)$ in Nakada and Mead (1965). The horizontal dotted line is $N_{\mathrm{e}}(L)$ in Tverskoy $(1964,1965,1968,1969)$. The plasmapause (the midpoint of the steep decline of $N_{\mathrm{e}}$ ) on the thick broken curve corresponds to $L=5.2$.

From Fig. 4 it is clear that for $L>4$ at $\mu>1.25 \mathrm{keV} \mathrm{nT}^{-1}$ the functions $F(\mu)$ almost does not depend on $L$. This is because the energy spectra of protons on $L>4$ at $\mu>1.25 \mathrm{keV} \mathrm{nT}^{-1}$ have similar power form in a variety of $L$ (see more about this in Kovtyukh, 1985, 1999).

The main contribution to the Coulomb losses of protons of the radiation belts make the cold plasma electrons (Tverskoy, 1964, 1965, 1968, 1969; Nakada and Mead, 1965; Schulz and Lanzerotti, 1974; Spjeldvik, 1977, Jentsch, 1984), the density of which, $N_{\mathrm{e}}$, is strongly dependent on $L$ (see Fig. 5).

Mean values $N_{\mathrm{e}}(L)$ near equatorial plane, averaged over MLT, were determined here by the empirical model of Ozhogin et al. (2012), created according to data of the IMAGE (the thick broken curve in Fig. 5), and also according to the results of Tarsai et al. (1988) based on measurements of the whistlers (the bright spots in Fig. 5). The results of the papers agree well with each other. The model of Carpenter and Anderson (1992) is also taken into account, created according to data of the ISEE-1 and ground-based data on whistlers (the dark dots in Fig. 5).

In the region of the plasmapause a value $N_{\mathrm{e}}$ varies by an order of magnitude within $\Delta L \sim 0.5$. The most reliable value is the location of plasmapause, averaged over MLT, obtained according to the CRRES: $L_{\mathrm{pp}}=(5.39 \pm 0.072)-$ $(0.382 \pm 0.019) \mathrm{Kp}_{\max }$, where $\mathrm{Kp_{ \operatorname {max } }}$ is the maximum value of $K p$ for the 24 hours preceding (Moldwin et al., 2002). In

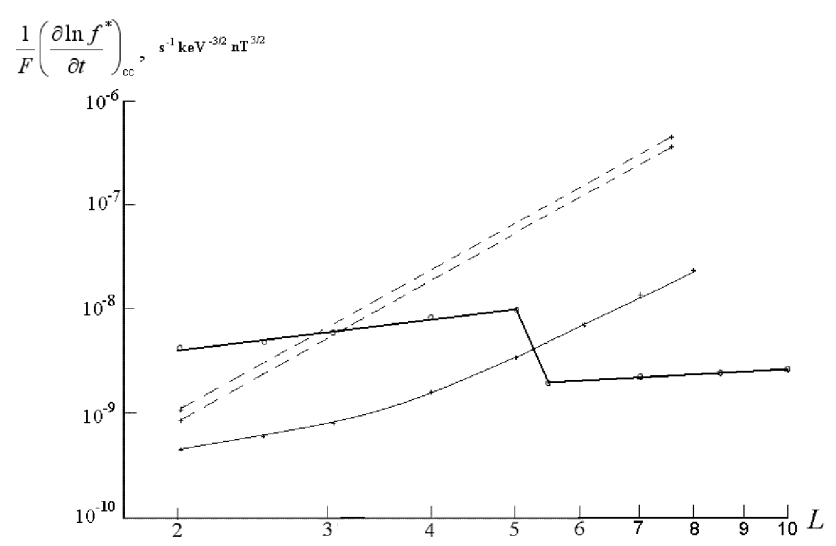

Figure 6. The radial dependences of the Coulomb losses of protons of the Earth's radiation belts, normalized to $f^{*}(L, \mu)$ and $F(L, \mu)$. The thick, thin and dotted curves correspond to the curves in Fig. 5.

Fig. 5, constructed for quiet periods, the plasmapause (the midpoint of the steep decline of $N_{\mathrm{e}}$ ) corresponds to $L \sim 5.2$.

Figure 5 shows also, for comparison, the functions $N_{\mathrm{e}}(L)$ used in the classical papers on modeling radial diffusion of particles of the Earth's radiation belts. Tverskoy (1964, $1965,1968,1969)$ assumed that $N_{\mathrm{e}}=10^{3} \mathrm{~cm}^{-3}$ and does not depend on $L$ (the dotted line in Fig. 5). Nakada and Mead (1965) assumed that $N_{\mathrm{e}}(L)=8 \cdot 10^{3} L^{-4}+50 \mathrm{~cm}^{-3}$ (the light line in Fig. 5), i.e. on $L>5$ the function flattens out and $N_{\mathrm{e}}$ is close to $50 \mathrm{~cm}^{-3}$.

Figure 5 shows that in Tverskoy $(1964,1965,1968,1969)$ the value $N_{\mathrm{e}}$ significantly exceeds the real average values on $L>3$ and underestimates them on $L<3$. Figure 5 shows also that in Nakada and Mead (1965) the values of $N_{\mathrm{e}}$ were underestimated by almost an order of magnitude on $L<5$ and greatly inflated on $L>5.5$.

The value of $N_{\mathrm{e}}$ decreases as $L^{-4.5}$ outside of the plasmapause (e.g., Jentsch, 1984; Carpenter and Anderson, 1992). Hence, according to Eq. (9), for energetic protons with identical $\mu$ outside of the plasmapause the rate of the Coulomb losses by free electrons is not dependent on $L$ (or this dependence is very weak).

Compared to the free electrons, atomic electrons make a much smaller contribution to the Coulomb losses of energetic protons. In the case of equal densities of the free and the bounded electrons, losses of protons on the bounded electrons is 12.5 times less (Cornwall, 1972; Schulz and Lanzerotti, 1974). In the core of the plasmasphere, this addition is only a few percent, but for the region of the plasmapause it is comparable to the Coulomb losses of protons by free electrons and increases with increasing $L$.

Compared to electrons, protons of the cold plasma makes a negligible contribution to the Coulomb losses of energetic protons (e.g., Schulz and Lanzerotti, 1974).

The results of our calculations of the rate of the Coulomb losses of protons of the radiation belts, $\left(\partial f^{*} / \partial t\right)_{\mathrm{cc}}$, normal- 
ized by functions $f^{*}(L, \mu)$ and $F(\mu)$, are shown in Fig. 6 by the thick curve. The curve is obtained by Eq. (11), in which the values of $N_{\mathrm{e}}(L)$ correspond to the thick curve in Fig. 5 . In the calculations electrons in atoms were also taken into account, the density of which was calculated by Eq. (6) and divided by the factor of 12.5. For comparison, Fig. 6 shows also the dotted curves corresponding to the values of $N_{\mathrm{e}}$ in Tverskoy $(1964,1965,1968,1969)$ and the thin curve corresponding to the values of $N_{\mathrm{e}}(L)$ in Nakada and Mead (1965).

From Fig. 6 it is seen that in Tverskoy $(1964,1965,1968$, 1969) the rates of Coulomb losses of protons of the Earth's radiation belts is underestimated on $L<2.8$ and highly inflated on $L>2.8$, and in Nakada and Mead (1965) they are very low inside and high outside the plasmasphere.

In the region of plasmapause errors of our calculations of the rates of the Coulomb losses of protons can be greater than in other regions. Furthermore, the model distribution of cold plasma, used in our calculations, is averaged over a very long time. They do not take into account the solarcyclic and seasonal variations of $N_{\mathrm{e}}(L)$ values. In the solar maximum $N_{\mathrm{e}}(L)$ is 1.3-1.5 times higher than in the minimum (e.g., Rasmussen and Schunk, 1990). Average values of $N_{\mathrm{e}}(L)$ are 1.5-2.2 times more in December than in June (e.g., Rasmussen and Schunk, 1990; Menk et al., 2012).

\section{Composition of Coulomb losses and charge exchange losses of protons}

Normalized rates of the Coulomb loss of proton, $-\left(\partial \ln f^{*} / \partial t\right)_{\mathrm{cc}}$, and the charge exchange rates, $-\left(\partial \ln f^{*} / \partial t\right)_{\mathrm{ce}}$, summarized, in accordance with Eq. (1), and thus we have full rates of the ionization losses of protons of the radiation belts: $-\left(\partial \ln f^{*} / \partial t\right)$. Figure 7 shows an example of such summation, for the protons with $\mu=0.7 \mathrm{keVnT}^{-1}\left(70 \mathrm{MeVG}^{-1}\right)$. In this figure, the continuous curves correspond to the charge exchange (upper curve) and the Coulomb losses, and the dotted curve is the result of their summation. The vertical cut on the dotted curve marks $L$, at which rate the Coulomb loss is equal to the charge exchange rate of the protons.

From Fig. 7 it is seen that in the narrow region the rate of the Coulomb and the charge exchange losses of protons are close to each other, and at smaller and larger $L$ they are diverging strongly. At small $L$ dominate the Coulomb losses, and at large $L$ dominate the charge exchange of protons. This is true for all protons the radiation belts with $\mu$ from 0.3 to $10 \mathrm{keV} \mathrm{nT}^{-1}$.

Final results of the calculations of the normalized rate of ionization losses of protons of the Earth's radiation belts with different values of $\mu$, obtained with modern models of the plasmasphere and the exosphere, are shown in Fig. 8. This figure takes into account and assimilates all the results of the calculations shown in Figs. 1-7. The numbers near the curves in Fig. 8 are given the corresponding values of $\mu$, from 0.3 to $10 \mathrm{keV} \mathrm{nT}^{-1}$. The vertical cuts on the curves marked the

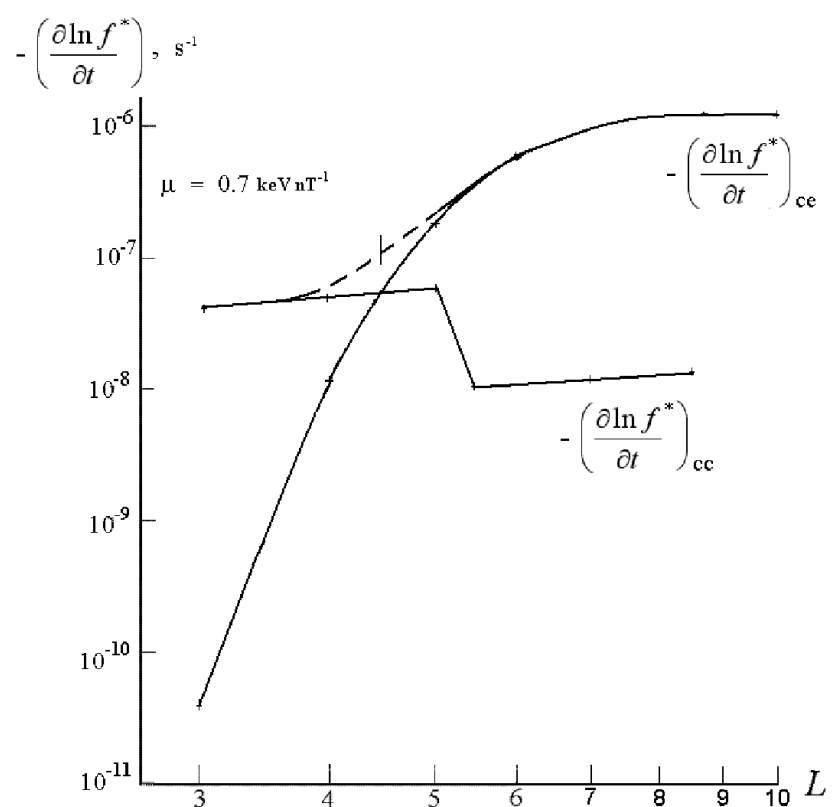

Figure 7. The example of summation of the rates of the Coulomb losses, $-\left(\partial \ln f^{*} / \partial t\right)_{\text {cc }}$, and losses on charge exchange, $-\left(\partial \ln f^{*} / \partial t\right)_{\mathrm{ce}}$, for protons with $\mu=0.7 \mathrm{keV} \mathrm{nT}^{-1}$.

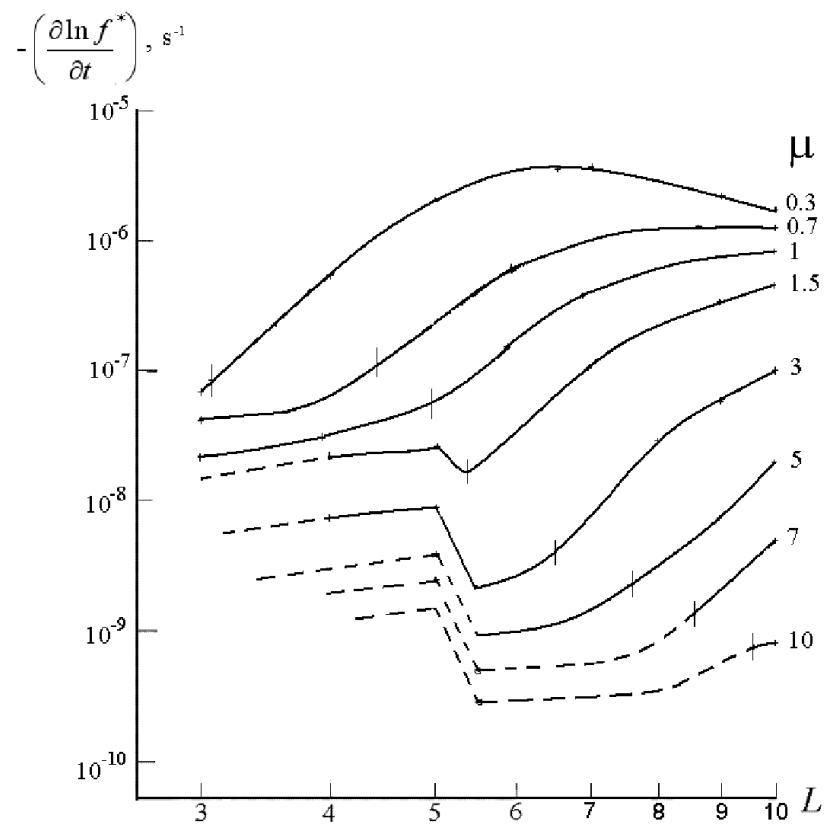

Figure 8. The radial dependence of the ionization losses of protons with $\mu$ from 0.3 to $10 \mathrm{keV} \mathrm{nT}^{-1}$, calculated on the modern models of the plasmasphere and the exosphere.

boundary where the rate of the Coulomb losses is equal to the rate of the charge exchange of protons. The dashed parts of curves in Fig. 8 obtained through extrapolation of the spectra at larger $\mu$ (see Fig. 3). 
In the constructing Fig. 8, we take into account that $\partial f^{*} / \partial \mu>0$ at $\mu<0.3-0.5 \mathrm{keV} \mathrm{nT}^{-1}$ (see Fig. 3). The Coulomb losses are subtracted from the losses caused by charge exchange of protons in this part of the spectrum.

From Fig. 8 it is seen that rates of ionization losses of protons of the Earth's radiation belts strongly depend on $\mu$ and decrease rapidly with increasing values of $\mu$, especially at high $L$. For protons with $\mu<1.2 \mathrm{keV} \mathrm{nT}^{-1}$ these rates monotonically decreases with decreasing $L$. In addition, for protons with $\mu>1.2 \mathrm{keV} \mathrm{nT}^{-1}$ this rate abruptly increases at the plasmapause.

Figure 9 shows the dependence from $\mu$ of the boundary where the rate of the Coulomb losses is equal to the rate of the charge exchange of protons. This dependence is well approximated by the function

$$
L_{\mathrm{b}}=4.71 \cdot \mu^{0.32} \text {, }
$$

where $[\mu]=\mathrm{keV} \mathrm{nT}^{-1}$. Coulomb losses of the trapped protons dominate at $L<L_{\mathrm{b}}(\mu)$, and at $L>L_{\mathrm{b}}(\mu)$ dominates charge exchange of protons with hydrogen atoms. Changes in the density of the plasmasphere in 2-3 times lead to slight variations in this relationship: $\delta L_{\mathrm{b}} \sim 0.1-0.3$. The value of $L_{\mathrm{b}}$ very weakly depends also on the position of the plasmapause.

In long quiet periods, outer regions of the plasmasphere is filled with cold plasma, and a sharp plasmapause on $L<7-8$, at least in the noon and evening sectors, does not exist (e.g., Clausen and Glassmeier, 2014; Takahashi et al., 2015; Halford et al., 2015). During the long quiet periods the plasmapause blurred and a cold plasma density gradually decreases from $\sim 200-300 \mathrm{~cm}^{-3}$ at $L=5$ and to $\sim 2 \mathrm{~cm}^{-3}$ at $L=8$. The effect leads to a degeneration of the local minimum in a radial dependency of the rates of ionization losses of protons with $\mu>1.2 \mathrm{keVnT}^{-1}$ (see Fig. 8). On the contrary, during magnetic storms and substorms this local minimum will deepen and be displaced, in line with displacement of plasmapause, to the smaller $L$. However, for future work we are only interested in measurements made during the quiet periods.

The errors of our calculations are associated mainly with deviations of the $N_{\mathrm{e}}(L)$ and $n_{\mathrm{H}}$ from the average values, and it can be estimated by varying these parameters within reasonable limits. The errors of our calculations can be several tens of percent in the region of plasmapause, at $L \sim 5-5.5$, for protons with $\mu \sim 1.5-10 \mathrm{keV} \mathrm{nT}^{-1}$. For other values of $L$ and $\mu$, the errors are much smaller and do not exceed $20 \%$.

\section{Comparison with results of other works}

First models of radial diffusion of the Earth's radiation belts of protons were created by Tverskoy $(1964,1965)$. Charge exchange of protons is not considered in the works, but Coulomb losses of protons are taken into account for the first time. The expression for Coulomb losses of protons obtained in Tverskoy $(1964,1965)$ on the basis of the well-known

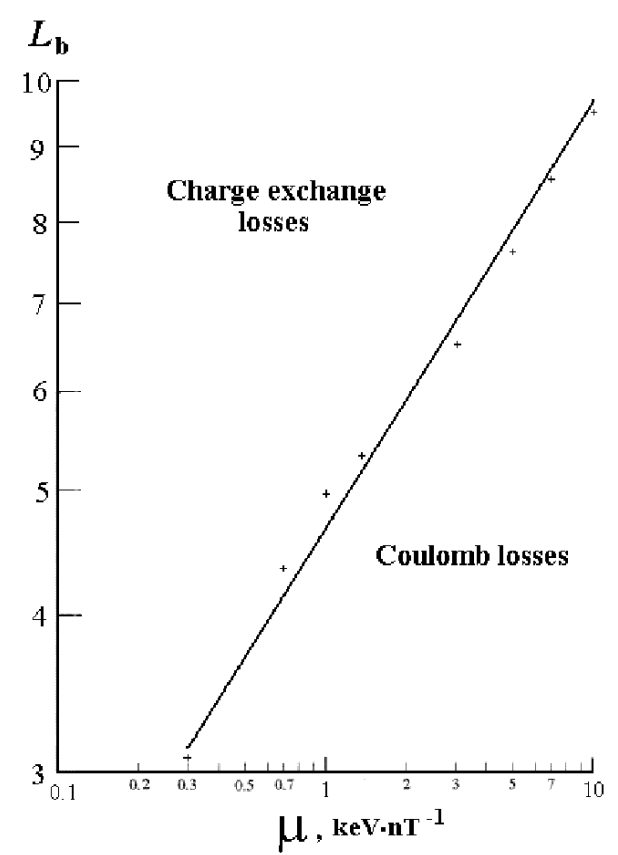

Figure 9. The dependence from $\mu$ of the boundary $L_{\mathrm{b}}$ where the rate of Coulomb losses is equal to the rate of the charge exchange of protons. This dependence is obtained from Fig. 8 (results of Fig. 8 shows the crosses). Thick line shows the power approximation of this dependence (Eq. 13).

Bethe-Bloch's formula. Taking here the notations and the dimensions of values, the expression has the following form:

$-\frac{1}{f^{*}}\left(\frac{\partial f^{*}}{\partial t}\right)_{\mathrm{cc}}=-3.79 \cdot 10^{-14} \cdot N_{\mathrm{e}} L^{9 / 2} F_{1}(\mu)$,

where $[t]=\mathrm{s}, N_{\mathrm{e}}(L)=10^{3} \mathrm{~cm}^{-3}$ (see Fig. 5) and

$$
\begin{aligned}
F_{1}(\mu) & =\frac{1}{f^{*}} \frac{\partial}{\partial \mu}\left(\frac{f^{*}}{\sqrt{\mu}}\right) \\
& =\frac{1}{f^{*} \sqrt{\mu}} \frac{\partial f^{*}}{\partial \mu}-\frac{1}{2 \mu^{3 / 2}}=F(\mu)-0.5 \mu^{-3 / 2} .
\end{aligned}
$$

The numerical coefficient in the right side of Eq. (14) is $20 \%$ less than the same coefficient in Eq. (11) because $\ln \Lambda=20$ was taken in Tverskoy $(1964,1965,1968)$. In Tverskoy (1969) the error was corrected: it was taken as $\ln \Lambda=25$, as in Eq. (11).

For real spectra of protons, the functions $F(\mu)$ and $F_{1}(\mu)$ differ by several tens of percent for $\mu>1 \mathrm{keV} \mathrm{nT}^{-1}$, but for $\mu<0.7 \mathrm{keV} \mathrm{nT}^{-1}$ they may differ by several times and they have a different sign (see Figs. 3 and 4). However in all particular calculations in Tverskoy $(1964,1965,1968)$ it is assumed that $F_{1}(\mu)=-1$.

The above also mentioned significant deviations assumed in Tverskoy $(1964,1965,1968,1969)$ model of a plasmasphere from the modern models $N_{\mathrm{e}}(L)$ and the associated 
large errors in calculations of the Coulomb losses of protons (see Figs. 5 and 6).

In (Nakada and Mead, 1965) for the Coulomb losses of protons the wrong Eq. (15) was also used. If you use the units taken in Nakada and Mead (1965), i.e. days instead of seconds, and $\mathrm{MeV} \mathrm{G}^{-1}$ instead of $\mathrm{keV} \mathrm{nT}^{-1}$, then the numerical coefficient in Eq. (14) will be equal to $3.27 \cdot 10^{-6}$. It is $8.6 \%$ less than the value of $3.55 \cdot 10^{-6}$ given in Nakada and Mead (1965). A more significant error of the calculations of Nakada and Mead (1965) related to underestimation of $N_{\mathrm{e}}(L)$ inside plasmasphere by about an order of magnitude and a strong overestimation of $N_{\mathrm{e}}$ outside the plasmasphere (see Figs. 5 and 6).

Nakada and Mead (1965) have also taken into account the charge exchange of protons with hydrogen atoms, but values of $n_{\mathrm{H}}$ near the equatorial plane were assumed to be equal to $7.35 \cdot 10^{3} L^{-5}$, i.e. $5.44 \cdot L^{3 / 2}$ times less than the values of $n_{\mathrm{H}}$ accepted in our calculations (see Eq. 6).

Further, in the works on the modeling of the protons of Earth's radiation belts (e.g., Cornwall, 1972; Claflin and White, 1974; Croley et al., 1976; Spjeldvik, 1977; Albert et al., 1998) Eqs. (9)-(11) were usually used for Coulomb losses. However, very different functions of $N_{\mathrm{e}}(L)$ were chosen in the works, and the function $F(\mu)$ is not always taken into account or this account has been pretty rough.

In Albert et al. (1998) the fluxes of protons with $E \sim 1-$ $100 \mathrm{MeV}$ for $L \leq 3$, measured on the CRRES, were modeled based on the theory of radial diffusion of particles. For rate of Coulomb losses of protons an expression similar to our Eq. (9) was used. It differs from Eq. (9) only a sign of an exponent for the induction $B(3 / 2$ instead of $-3 / 2)$, but it is apparently just a misprint.

According to the results of the calculations shown in Fig. 3 in Albert et al. (1998), a ratio of rates of Coulomb losses of protons with $\mu=1$ and $10 \mathrm{keV} \mathrm{nT}^{-1}$ (100 and $1000 \mathrm{MeV} \mathrm{G}^{-1}$ ) is equal to $\sim 110$. The ratio is equal to the ratio of corresponding values of the function $F(\mu)$ on $L=3$ (see Eq. 10).

Data from the ISEE-1 and Explorer-45, used here, do not allow to construct functions $f^{*}(\mu)$ for protons with $\mu=1-$ $10 \mathrm{keV} \mathrm{nT}^{-1}$ on $L \leq 3$. However, you can build the functions $f^{*}(\mu)$ using the CRRES data shown on Fig. 4 in Albert et al. (1998). For quiet periods the functions $f^{*}(\mu)$ for protons with $\alpha_{0}=90^{\circ}$ on $L=2$ and 3 , constructed according to the CRRES data, are shown in Fig. 3 by a dotted line. These functions are normalized and linked to the functions $f^{*}(\mu)$ constructed according to the ISEE-1 data. According to the CRRES data, is shown in Fig. 3, on $L=3$ the ratio of $F(\mu)$ values under $\mu=1$ and $10 \mathrm{keV} \mathrm{nT}^{-1}$ equal $\sim 40-60$, i.e. in $\sim 2.2$ times less than the corresponding value $(\sim 110)$ calculated in Albert et al. (1998). Thus, in Albert et al. (1998) a dependence of the rate of Coulomb losses of protons belts from an inclination of the spectrum was considered very rough.

\section{Discussion and conclusion}

For modern models of the plasmasphere and the exosphere and the known cross sections for Coulomb collisions and charge exchange of protons we calculated and presented here the radial profiles of rates of the ionization losses of protons of the Earth's radiation belts, for various values of $\mu$.

In the qualitative analysis of radial diffusion of protons of the Earth's radiation belts ionization losses are considered generally as a function of the particle energy. Considering the losses depending on $\mu$ allows one to look at them with a new perspective and detect unexpected patterns.

Thus, in many articles and books it is claimed that with decreasing $L$ the rate of ionization losses of protons increases due to increased density of the cold plasma and the atoms of the exosphere. This applies to protons with a given energy value. However, the particles of the radiation belts migrated across $L$ with conservation of the first adiabatic invariant, $\mu$, which is reflected in a diffusion equation and responds to the growth of the particle energy with decrease in $L$ as a result of the betatron acceleration. Outside the equatorial plane we must also consider the conservation of the second adiabatic invariant, $K$, which also increases an energy of particles with decreasing $L$. Therefore, as can be seen from Fig. 8, the rate of ionization losses of protons with constant $\mu$ tends to decrease with decreasing $L$. The dependences have a negative gradient only in a narrow region $(\Delta L \sim 0.5)$ in the region of plasmapause and only for protons with $\mu>1.2 \mathrm{keV} \mathrm{nT}^{-1}$.

A radial dependence of a cold plasma density smoothed in fairly long quiet periods, and a local minimum in the dependences of protons ionization loss rates from $L$ may degenerate. On the contrary, during magnetic storms and substorms this local minimum will deepen and be displaced, in line with displacement of plasmapause, to the smaller $L$; at $L<3.5$ and on $L>6$ the rates of ionization losses of protons will vary slightly.

These findings are very important for understanding the results of a numerical simulation of distributions of protons of the Earth's radiation belts.

Our results also show that for radiation belts of the Earth

- the charge exchange of protons with atoms is much more important, than it was previously accepted, for a formation of an equilibrium belt of protons and dominates over the Coulomb losses of protons at $E<300-$ $400 \mathrm{keV}$ (this also applies to other, more heavy ions);

- Coulomb losses depend strongly on $\mu$ of protons and the shape of their energy spectra; they abruptly changed in the region of plasmapause and weakly depend on $L$ outside the narrow region;

- in some domains $\{\mu, L\}$ spectrum of protons can have a local maximum and where $\partial f^{*} / \partial \mu>0$ of protons change their sign; in the domains the losses are sub- 
tracted from the losses of protons to charge exchange; this effect simulates a local source of particles;

- a full rates of ionization losses of protons depends strongly on $\mu$ and decreases rapidly with increasing $\mu$, especially at high $L$.

The results of our calculations of the rate of ionization losses of protons of the Earth's radiation belts can serve as the basis for calculation of functions $D_{L L}(L, \mu)$ on the experimental data obtained near the equatorial plane in quiet and weakly disturbed periods. The results of these calculations will be presented in the next papers of the author. 


\section{Appendix A: Calculations of the functions $f^{*}(L, \mu)$}

For a more exact binding differential fluxes to an energy of the particles, calculations of the functions $f^{*}(L, \mu)$ were carried out in four stages. In the first stage the fluxes were attached to the arithmetic mean values of the energy in the channels, and on these points an energy spectra of protons $j(E)$ on different $L$ was constructed. In the second stage the spectra were approximated by power functions $\left(j \propto E^{-\gamma}\right)$, for each an energy channel, and values of the exponents $\gamma$ were calculated (for different channels and different $L$ they are different). In the third stage the values of $\gamma$ were used to find the energy $\widetilde{E}$ by the following expression:

$$
\begin{aligned}
j(\widetilde{E}) & =j_{0} \widetilde{E}^{-\gamma}=\frac{1}{\Delta E} \int_{E_{1}}^{E_{2}} j_{0} E^{-\gamma} \mathrm{d} E \\
& =\frac{j_{0}}{\Delta E} \cdot \frac{E_{1}^{-\gamma+1}-E_{2}^{-\gamma+1}}{\gamma-1},
\end{aligned}
$$

where $j_{0}$ is a normalizing factor of the spectra, $\Delta E$ is the channel width, $E_{1}$ and $E_{2}$ are the lower and upper bounds. For each a channel the value $\widetilde{E}$ depends on the $L$. From Eq. (A1) we find:
$\widetilde{E}=\left[\frac{\Delta E(\gamma-1)}{E_{1}^{-\gamma+1}-E_{2}^{-\gamma+1}}\right]^{1 / \gamma}$.

Equation (A2) is valid at $\gamma>1$. For $\gamma=1$

$\widetilde{E}=\frac{\Delta E}{\ln \left(E_{2} / E_{1}\right)}$,

and for $\gamma<1$ (except for $\gamma=0$ )

$\widetilde{E}=\left[\frac{\Delta E(1-\gamma)}{E_{2}^{-\gamma+1}-E_{1}^{-\gamma+1}}\right]^{1 / \gamma}$.

For $\gamma=0$, as for $\gamma=-1, \widetilde{E}=\left(E_{1}+E_{2}\right) / 2=\bar{E}$.

The wider a channel, the greater a correction to the energy $\widetilde{E}$ (except for $\gamma=0$ and $\gamma=-1$ ). The most significant corrections refer to the last four channels of proton spectrometer on the ISEE-1.

In the fourth stage calculating of $f^{*}(L, \mu)$ fluxes measured in each channel was divided by the corresponding values of $\widetilde{E}$ and attached to $\mu=\widetilde{E} / B(L)$. 
Acknowledgements. The author would like to thank P. Kollmann (Max Planck Institute for Solar System Research) for very important and fruitful comments and proposals to the paper, and E. Roussos (Max Planck Institute for Solar System Research) for redaction of the paper. The author thanks Kyoto World Data Center for Geomagnetism for providing the Dst indices.

The topical editor E. Roussos thanks P. Kollmann and F. Soraas for help in evaluating this paper.

\section{References}

Albert, J. M., Ginet, G. P., and Gussenhoven, M. S.: CRRES observations of radiation belt protons. 1. Data overview and steady state radial diffusion, J. Geophys. Res., 103, 9261-9273, doi:10.1029/97jA02869, 1998.

Alinejad, N. and Armstrong, T. P.: Radial diffusion of geomagnetically trapped protons observed by the Galileo Energetic Particle Detector, J. Geophys. Res., 111, A09209, doi:10.1029/2005jA011040, 2006.

Carpenter, D. L. and Anderson, R. R.: An ISEE/whistler model of equatorial electron density in the magnetosphere, J. Geophys. Res., 97, 1097-1108, doi:10.1029/91jA01548, 1992.

Claflin, E. S.: Charge-exchange cross sections for hydrogen and helium ions incident on atomic hydrogen: 1 to $1000 \mathrm{keV}$, Rep. TR0059(6260-20)-1, The Aerospace Corporation, El Segundo, CA, USA, 1970.

Claflin, E. S. and White, R. S.: A study of equatorial inner belt protons from 2 to $200 \mathrm{MeV}$, J. Geophys. Res., 79, 959-965, doi:10.1029/JA079i007p00959, 1974.

Clausen, L. B. N. and Glassmeier, K.-H.: Enhancement of ultralow frequency wave amplitudes at the plasmapause, J. Geophys. Res.-Space, 119, 9113-9124, doi:10.1002/2014JA020072, 2014.

Cornwall, J. M.: Radial diffusion of ionized helium and protons: A probe for magnetosphere dynamics, J. Geophys. Res., 77, 17561770, doi:10.1029/JA077i010p01756, 1972.

Cowley, S. W. H.: Pitch angle dependence of the charge-exchange lifetime of ring current ions, Planet. Space Sci., 25, 385-393, doi:10.1016/0032-0633(77)90054-X, 1977.

Croley Jr., D. R., Schulz, M., and Blake, J. B.: Radial diffusion of inner-zone protons: Observations and variational analysis, J. Geophys. Res., 81, 585-594, doi:10.1029/JA081i004p00585, 1976.

Fritz, T. A. and Spjeldvik, W. N.: Steady-state observations of geomagnetically trapped energetic heavy ions and their implications for theory, Planet. Space Sci., 29, 1169-1193, doi:10.1016/00320633(81)90123-9, 1981.

Halford, A. J., Fraser, B. J., and Morley, S. K.: EMIC waves and plasmaspheric and plume density: CRRES results, J. Geophys. Res.-Space, 120, 1974-1992, doi:10.1002/2014JA020338, 2015.

Jentsch, V.: The radial distribution of radiation belt protons: Approximate solution of the steady state transport equation at arbitrary pitch angle, J. Geophys. Res., 89, 1527-1539, doi:10.1029/JA089iA03p01527, 1984.

Kovtyukh, A. S.: On the form of energy spectrum of protons of the Earth's radiation belts and the mechanisms of its formation, Geomagn. Aeron., 25, 886-892, 1985.
Kovtyukh, A. S.: Solar-cycle variations of invariant parameters of ion energy spectra of the Earth's radiation belts, Cosmic Res., 37, 53-64, 1999.

Lindsay, B. G. and Stebbings, R. F.: Charge transfer cross sections for energetic neutral atom data analysis, J. Geophys. Res., 110, A12213, doi.10.1029/2005JA011298, 2005.

Lyons, L. R. and Williams, D. J.: Quantitative Aspects of Magnetospheric Physics, D. Reidel, Norwell, MA, USA, 1984.

Menk, F. W., Ables, S. T., Grew, R. S., Clilverd, M. A., and Sandel, B. R.: The annual and longitudinal variations in plasmaspheric ion density, J. Geophys. Res., 117, A03215, doi:10.1029/2011jA017071, 2012.

Moldwin, M. B., Downward, L., Rassoul, H. K., Amin, R., and Anderson, R. R.: A new model of the location of the plasmapause: CRRES results, J. Geophys. Res., 107, 1339, doi:10.1029/2001jA009211, 2002.

Nakada, M. P. and Mead, G. D.: Diffusion of protons in the outer radiation belt, J. Geophys. Res., 70, 4777-4791, doi:10.1029/JZ070i019p04777, 1965.

Østgaard, N., Mende, S. B., Frey, H. U., Gladstone, G. R., and Lauche, H.: Neutral hydrogen density profiles derived from geocoronal imaging, J. Geophys. Res., 108, 1300, doi:10.1029/2002jA009749, 2003.

Ozhogin, P., Tu, J., Song, P., and Reinisch, B. W.: Field-aligned distribution of the plasmaspheric electron density: An empirical model derived from the IMAGE RPI measurements, J. Geophys. Res., 117, A06225, doi:10.1029/2011jA017330, 2012.

Rasmussen, C. E. and Schunk, R. W.: A three-dimensional timedependent model of the plasmasphere, J. Geophys. Res., 95, 6133-6144, doi:10.1029/jA095iA05p06133, 1990.

Roederer, J. G.: Dynamics of Geomagnetically Trapped Radiation, Springer, New York, USA, 1970.

Schulz, M. and Lanzerotti, L. J.: Particle Diffusion in the Radiation Belts, Springer, New York, USA, 1974.

Spjeldvik, W. N.: Equilibrium structure of equatorially mirroring belt protons, J. Geophys. Res., 82, 2801-2808, doi:10.1029/JA082i019p02801, 1977.

Takahashi, K., Denton, R. E., Kurth, W., Kletzing, C., Wygant, J., Bonnell, J., Dai, L., Min, K., Smith, C. W., and MacDowall, R.: Externally driven plasmaspheric ULF waves observed by the Van Allen Probes, J. Geophys. Res., 120, 526-552, doi:10.1002/2014JA020373, 2015.

Tarsai, G., Szemerédy, P., and Hegymegi, L.: Average electron density profiles in the plasmasphere between $L=1.4$ and 3.2 deduced from whistlers, J. Atmos. Sol.-Terr. Phy., 50, 607-611, doi:10.1016/0021-9169(88)90058-X, 1988.

Tverskoy, B. A.: Dynamics of the radiation belts of the Earth, Geomagn. Aeron., 4, 351-368, 1964.

Tverskoy, B. A.: Transport and acceleration of charged particles in the Earth's magnetosphere, Geomagn. Aeron., 5, 517-530, 1965.

Tverskoy, B. A.: Dynamics of the Earth's Radiation Belts, Nauka, Moscow, Russia, 1968.

Tverskoy, B. A.: Main mechanisms in formation of the Earth's radiation belts, Rev. Geophys., 7, 219-232, doi:10.1029/RG007i001p00219, 1969.

West, H. I., Buck, R. M., and Davidson, G. T.: The dynamics of energetic electrons in the Earth's outer radiation belt during 1968 as observed by the Lawrence Livermore National Labora- 
tory's spectrometer on Ogo 5, J. Geophys. Res., 86, 2111-2142, doi:10.1029/JA086iA04p02111, 1981.

Williams, D. J.: Phase space variations of near equatorially mirroring ring current ions, J. Geophys. Res., 86, 189-194, doi:10.1029/jA086iA01p00189, 1981.
Zoennchen, J. H., Nass, U., and Fahr, H. J.: Exospheric hydrogen density distributions for equinox and summer solstice observed with TWINS1/2 during solar minimum, Ann. Geophys., 31, 513527, doi:10.5194/angeo-31-513-2013, 2013. 